\title{
Cardiac TRPV1 afferent signaling promotes arrhythmogenic ventricular remodeling after myocardial infarction
}

\author{
Koji Yoshie, ${ }^{1,2}$ Pradeep S. Rajendran, ${ }^{1,2}$ Louis Massoud, ${ }^{1,2}$ Janki Mistry, ${ }^{1,2}$ M. Amer Swid, ${ }^{1,2}$ \\ Xiaohui Wu, ${ }^{2}$ Tamer Sallam, ${ }^{2,3}$ Rui Zhang, ${ }^{4}$ Joshua I. Goldhaber, ${ }^{4,5}$ Siamak Salavatian, ${ }^{1,2}$ \\ and Olujimi A. Ajijola, \\ ${ }^{1}$ Cardiac Arrhythmia Center and Neurocardiology Research Program, ${ }^{2}$ Division of Cardiology, Department of Medicine, and \\ ${ }^{3}$ Molecular Biology Institute, UCLA, Los Angeles, California, USA. ${ }^{4}$ Cedars Sinai Heart Institute, Los Angeles, California, USA. \\ ${ }^{5}$ Molecular, Cellular and Integrative Physiology Interdepartmental Program, UCLA, Los Angeles, California, USA.
}

\begin{abstract}
Chronic sympathoexcitation is implicated in ventricular arrhythmogenesis (VAs) following myocardial infarction (MI), but the critical neural pathways involved are not well understood. Cardiac adrenergic function is partly regulated by sympathetic afferent reflexes, transduced by spinal afferent fibers expressing the transient receptor potential cation subfamily $\mathbf{V}$ member 1 (TRPV1) channel. The role of chronic TRPV1 afferent signaling in VAs is not known. We hypothesized that persistent TRPV1 afferent neurotransmission promotes VAs after MI. Using epicardial resiniferatoxin (RTX) to deplete cardiac TRPV1-expressing fibers, we dissected the role of this neural circuit in VAs after chronic $\mathrm{MI}$ in a porcine model. We examined the underlying mechanisms using molecular approaches, IHC, in vitro and in vivo cardiac electrophysiology, and simultaneous cardioneural mapping. Epicardial RTX depleted cardiac TRPV1 afferent fibers and abolished functional responses to TRPV1 agonists. Ventricular tachycardia/fibrillation (VT/ VF) was readily inducible in MI subjects by programmed electrical stimulation or cesium chloride administration; however, TRPV1 afferent depletion prevented VT/VF induced by either method. Mechanistically, TRPV1 afferent depletion did not alter cardiomyocyte action potentials and calcium transients, the expression of ion channels, or calcium handling proteins. However, it attenuated fibrosis and mitigated electrical instability in the scar border zone. In vivo recordings of cardiovascular-related stellate ganglion neurons (SCNs) revealed that MI enhances SGN function and disrupts integrated neural processing. Depleting TRPV1 afferents normalized these processes. Taken together, these data indicate that, after MI, TRPV1 afferent-induced adrenergic dysfunction promotes fibrosis and adverse cardiac remodeling, and it worsens border zone electrical heterogeneity, resulting in electrically unstable ventricular myocardium. We propose targeting TRPV1-expressing afferent to reduce VT/VF following MI.
\end{abstract}

Conflict of interest: OAA and PSR report having equity in a startup (NeuCures)

Copyright: (ㄷ) 2020, American Society for Clinical Investigation.

Submitted: September 4, 2018

Accepted: December 12, 2019

Published: January 30, 2020.

Reference information: /CI Insight. 2020:5(2):e124477.

https://doi.org/10.1172/jci.

insight.124477.

\section{Introduction}

Cardiac adrenergic activation (sympathoexcitation) after myocardial infarction (MI) is a central driver of ventricular arrhythmogenesis (VAs) $(1,2)$. Pharmacologic adrenergic receptor (AR) antagonism and neurohormonal blockade form the basis of clinical therapy (3) after MI; however, current therapies are inadequate in preventing VAs.

The cardiac sympathetic afferent reflex (CSAR), which mediates the transduction of and sympathetic response to acute MI, contributes to the initiation and persistence of adrenergic activation (1, 4-6). The CSAR is enhanced in chronically injured and failing hearts (7). The afferent limb of the CSAR is mediated partly by fibers expressing the transient receptor potential cation subfamily V member 1 (TRPV1) channel (8), activated by heat, several metabolites generated by ischemia (e.g., bradykinin), and capsaicin. TRPV1 afferent signaling is also implicated in chronic pain $(9,10)$. Chronic cardiac sympathoexcitation driven by persistent TRPV1 afferent signaling promotes adverse remodeling in rodents (11). However, its role in arrhythmogenesis has not been explored. Furthermore, the translational potential of this paradigm in the treatment of progressive cardiac dysfunction after MI is unknown. 
To address this, we devised a strategy for cardiac-restricted targeting of TRPV1 afferents by transient percutaneous epicardial application of resiniferatoxin (RTX) (12), a potent, selective, and irreversible activator of the TRPV1 channel that depletes fibers containing the channel. RTX was applied at the time of MI induction in pigs. Terminal experimentation was performed 4 weeks later in the following groups: control, MI treated with RTX (MI + RTX), and untreated MI subjects.

Here, we demonstrate a substantial reduction in VAs in MI + RTX pigs compared with MI pigs, mediated by amelioration of scar border zone (BZ) fibrosis and sympathetic remodeling that converge to promote electrically unstable myocardium, and VAs. These data suggest that percutaneous epicardial modulation of TRPV1 sensory afferents is a viable upstream target to mitigate chronic cardiac sympathoexcitation and its deleterious effects.

\section{Results}

Epicardial RTX application induces degeneration of TRPV1 afferent fibers. To target cardiac-specific TRPV1-expressing afferent fibers (Figure 1A), we utilized a percutaneous approach to transiently apply RTX (12.5 $\mu \mathrm{g} / \mathrm{mL}$; Supplemental Methods) to the epicardium in MI subjects. We previously established the feasibility of this approach in normal porcine (12). Four weeks later, depletion of calcitonin gene-related peptide (CGRP), a marker of afferent fibers that colocalize with TRPV1 (13), was confirmed by epicardial IHC (Figure 1, B and C). Adrenergic fibers labeled by tyrosine hydroxylase (TH) immunoreactivity were not affected. Depletion of TRPV1 afferent fibers was also demonstrated functionally using TRPV1 agonists capsaicin (Figure 1, D and E) and bradykinin (Figure 1F). These activators normally result in robust adrenergic-induced hemodynamic responses but were abolished following RTX treatment. Additionally, immunohistochemical studies of dorsal root ganglia (DRG) from MI + RTX compared with control and MI subjects demonstrated regions of axon loss but no differences in DRG soma size (Supplemental Figure 1, A-C; supplemental material available online with this article; https://doi.org/10.1172/jci.insight.124477DS1).

Given the small diameter of C- and A $\delta$-axons that characterize TRPV1 afferents (14), selective loss of TRPV1 neurons would be expected to bias the distribution of DRG soma size toward larger neurons. The absence of this finding, in the presence of loss of axons, indicates that epicardial RTX application caused axonal degeneration of TRPV1 afferents and not significant elimination of sensory afferent soma in the DRG. Both chemical (TRPV1-expressing) and multimodal (mechanical and chemical) cardiac afferents were likely impacted by RTX, given the expression of TRPV1 in these fibers.

Cardiac TRPV1 afferent depletion reduces VAs after MI. To determine whether depletion of cardiac TRPV1 afferents reduces VAs after MI, we performed programmed extrastimulation (PES) from a right ventricular endocardial catheter 4 weeks after anteroseptal infarct in MI and MI + RTX subjects. Ventricular tachycardia (VT) or ventricular fibrillation (VF) was induced in 12 of $13 \mathrm{MI}$ subjects (92\%) but only in 1 of $7 \mathrm{MI}+$ RTX subjects $(14 \%)(P=0.005$; Figure 2, A and B). The ease of inducibility (i.e., degree of aggressiveness of the PES protocol) was quantified as the arrhythmogenicity index (AI), which was higher when fewer ventricular extrastimuli were required for induction. MI subjects compared with MI + RTX showed greater AI (Figure 2, C-E).

Arrhythmogenesis was also assessed by bolus i.v. administration of cesium chloride $(\mathrm{CsCl})$, a nonspecific potassium channel antagonist that transiently elicits afterdepolarization-induced triggered activity (15) (Supplemental Methods). Premature ventricular contractions (PVCs), as well as the number and length of nonsustained VT (NSVT) episodes, were reduced in MI + RTX (Figure 2, F-I) compared with MI.

In summary, these findings show that VAs, assessed by independent provocations, are significantly reduced by depletion of cardiac TRPV1 afferents.

TRPV1 afferent depletion does not mediate its antiarrhythmic effects by altering cardiomyocyte electrophysiology. Next, to determine the mechanisms by which cardiac TRPV1 afferent depletion reduced VAs after MI, we examined the expression of ion channels causative of VAs (16) in the scar BZ, where postinfarct VAs typically originate (17). Expression of SCN5A $\left(\mathrm{I}_{\mathrm{NA}}\right), \operatorname{KCND} 2\left(\mathrm{I}_{\mathrm{to}}\right), \operatorname{HERG}\left(\mathrm{I}_{\mathrm{kr}}\right), \mathrm{KCNQ} 1\left(\mathrm{I}_{\mathrm{ks}}\right)$, and $\mathrm{KCNJ} 4$ $\left(\mathrm{I}_{\mathrm{k} 1}\right)$ was not different (Supplemental Figure 2A) among control, MI, and MI + RTX subjects. Additionally, since $\beta$-adrenergic signaling modulates ion channel function, we examined and found no differences in $\beta_{1} \mathrm{AR}$ mRNA expression, protein levels, or functional responses to sympathetic stimulation (Supplemental Figure 2B) elicited by right stellate ganglion stimulation (RSGS).

Although expression of ion channels was not different, alterations in ion channel function by cardiac TRPV1 afferent depletion may underlie the protection from VAs. To examine this possibility, 
A

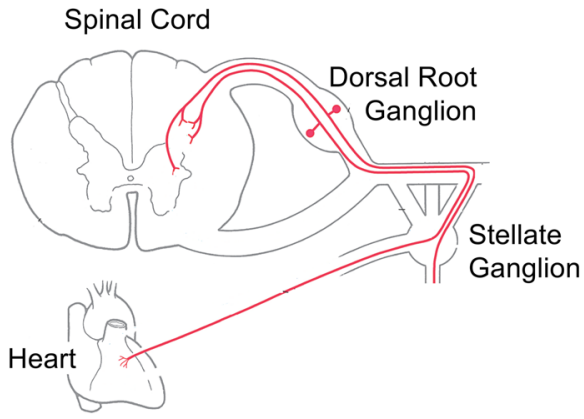

C

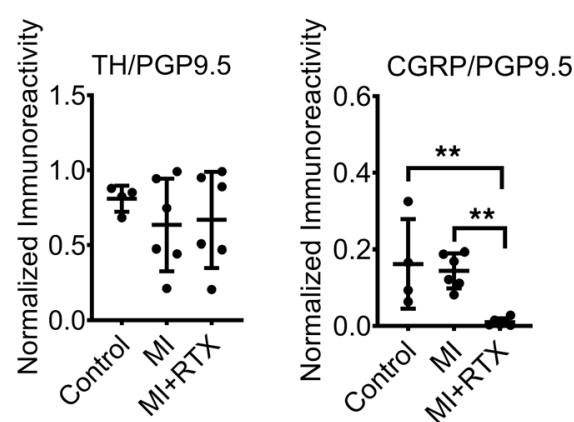

B

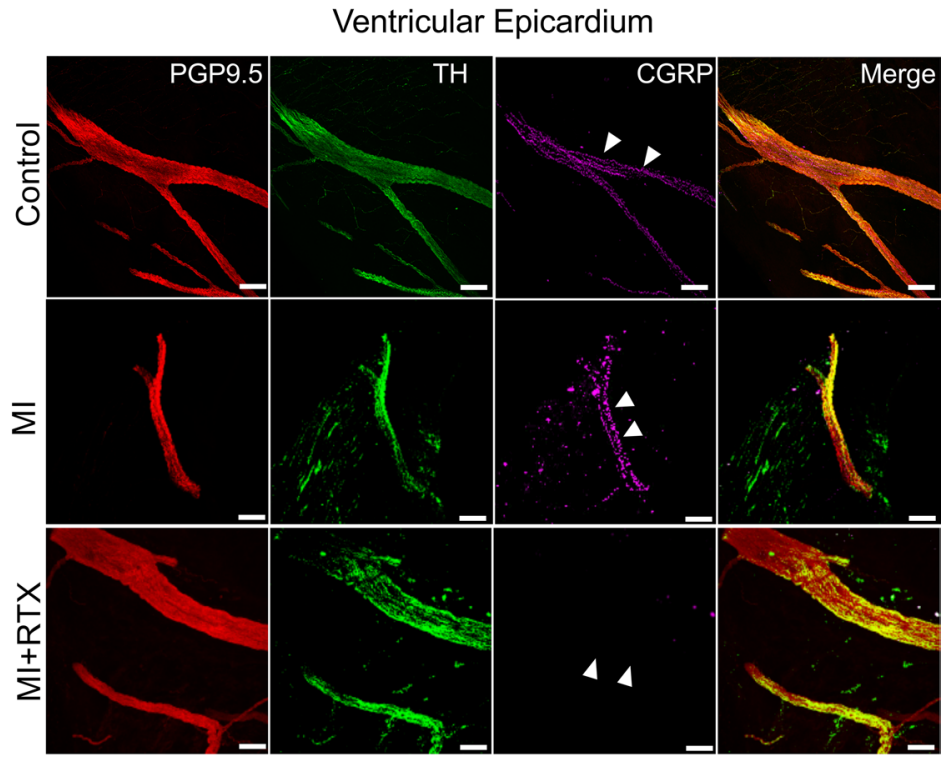

D

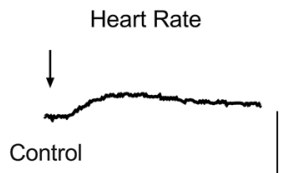

LV Pressure
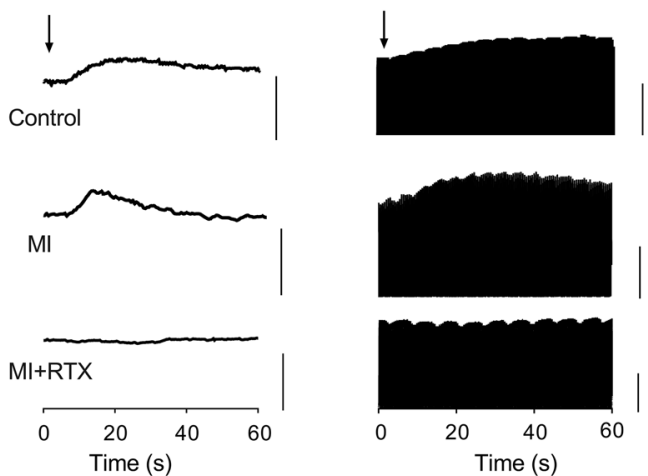

E

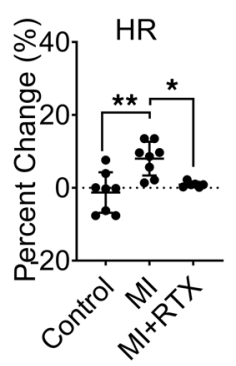

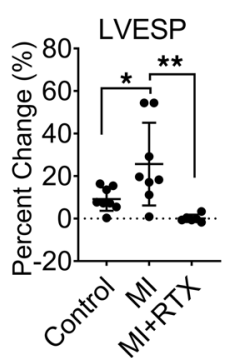

Capsaicin $20 \mu \mathrm{g} / \mathrm{ml}$
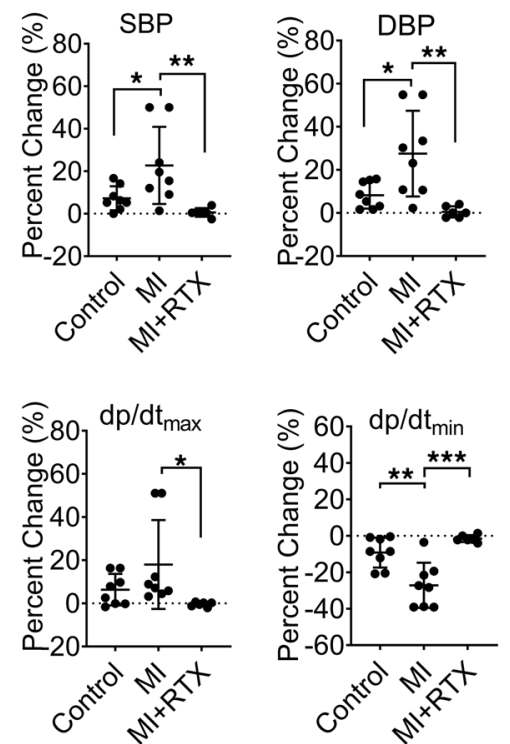

F Bradykinin $20 \mu \mathrm{g} / \mathrm{ml}$
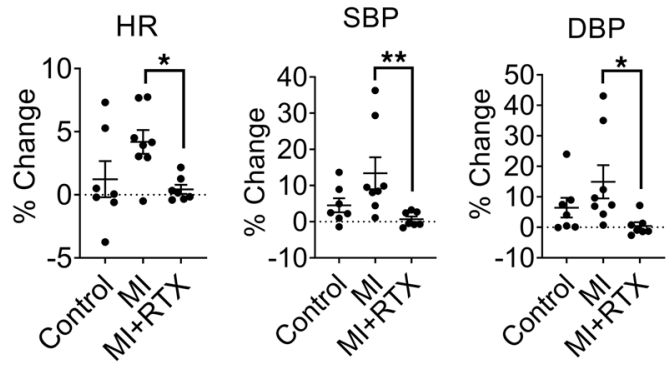

LVESP
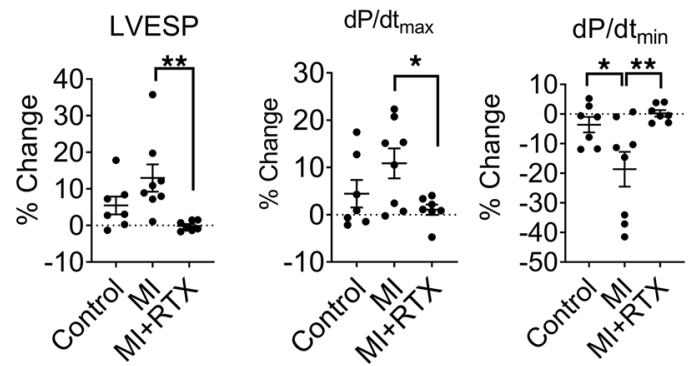
Figure 1. Chemical depletion of cardiac TRPV1 afferents by resiniferatoxin. (A) Schematic representation of the central projection of cardiac sympathetic afferent fibers. (B) Representative images of immunoreactivity (IR) to protein gene product 9.5 (PGP9.5), a panneuronal marker; tyrosine hydroxylase (TH, adrenergic marker); and calcitonin gene-related peptide (CGRP), a sensory peptide coexpressed by most TRPV1 afferents, are shown in LV epicardium of control, myocardial infarction (MI), and resiniferatoxin-treated (RTX-treated) MI subjects (MI + RTX). Scale bar: $50 \mu \mathrm{m}$. (C) Relative to nerve fibers seen, RTX depleted sensory but not adrenergic fibers ( $n=4-6 /$ group). (D-F) Epicardial depletion of TRPV1 afferents is further evidenced functionally by a lack of response to epicardial application of TRPV1 agonists, capsaicin ( $\mathbf{D}$ and $\mathbf{E})$ and bradykinin (F). Arrow in $\mathbf{D}$ indicates time of capsaicin application. Representative hemodynamic tracings of responses to capsaicin $20 \mu \mathrm{g} / \mathrm{mL}$ are shown in $\mathbf{D}$. MI subjects showed a sensitization of TRPV1 afferents compared with controls; however, responses were abolished in RTX-treated subjects. $n=6-8 /$ group. ${ }^{*} P<0.05 ;{ }^{* *} P<0.01$; ${ }^{* *} P<0.001$ by Kruskall-Wallis test.

we recorded action potentials (APs) from scar BZ cardiomyocytes isolated from MI and MI + RTX subjects. We confirmed that isolated cells from MI and MI + RTX subjects exhibited no morphological differences, since RTX was applied to the epicardium directly (Supplemental Figure 3, A and B). The APs of BZ cardiomyocytes from MI and MI + RTX subjects showed no differences in resting membrane potential, AP amplitude, upstroke velocity $\left(\mathrm{dV} / \mathrm{dt}_{\max }\right)$, and duration (AP duration-90 $\left[\mathrm{APD}_{90}\right]$ and $\mathrm{APD}_{50}$ ) (Figure $3, \mathrm{~A}-\mathrm{F}$ ).

To determine whether calcium handling, an accepted mechanism for arrhythmogenesis when dysfunctional (18), was involved in the reduced arrhythmogenic potential observed in MI + RTX animals, we investigated $\mathrm{Ca}^{2+}$ transients in cardiomyocytes loaded with the $\mathrm{Ca}^{2+}$-sensitive indicator Fluo-4. We found no significant differences in the kinetics of calcium release (time to peak) and reuptake (half maximal recovery $\left[\tau_{50}\right]$ ), as shown in Figure 3, G-I. Similarly, there were no differences in expression of the L-type calcium channel or other calcium handling proteins, as determined by RNA sequencing (RNA-seq) (Supplemental Figure 4A) and quantitative PCR (qPCR) (Supplemental Figure 4B).

These data indicate that modulation of cardiomyocyte AP and calcium handling do not underlie the antiarrhythmic protection conferred by cardiac TRPV1 afferent depletion following MI. It is possible that TRPV1 afferent depletion is associated with changes in the functional regulation of ion channels or calcium handling proteins; however, if these changes exist, they do not appear to translate to the cardiomyocyte $\mathrm{AP}$ or $\mathrm{Ca}^{2+}$ transient properties.

BZ electrical heterogeneity, fibrosis, and altered connexin distribution are attenuated by TRPV1 afferent depletion. The critical dependence of VAs on structural abnormalities that permit slowed and heterogeneous electrical propagation in the BZ of infarcted hearts is well established (17). To determine whether chronic TRPV1 afferent depletion exerts its protective effects by ameliorating cardiac structural abnormalities following MI, we quantified fibrosis, a determinant of electrical propagation, and performed high-resolution cardiac electrical mapping of the BZ in MI and MI + RTX subjects.

Trichrome staining of BZ myocardium demonstrated a substantial reduction in transmural fibrosis and wall thinning in MI + RTX versus MI subjects (Figure 4, A and B) in the scar BZ but not in remote myocardium. Next, we turned to RNA-seq of myocardium from the scar BZ to profile the expression of key proteins known to underlie collagen deposition following MI. As shown in Figure 4C, several genes involved in the regulation of collagen deposition after MI were differentially expressed in MI + RTX myocardium compared with MI, suggesting that several pathways key to infarct-related remodeling are impacted by cardiac TRPV1 afferent depletion.

To examine whether the reduction in fibrosis was of functional significance, we performed electrical mapping of the scar BZ as previously published (19). These studies demonstrated that electrical activation in scar BZ appeared slower and more heterogeneous in MI subjects, compared with MI + RTX. Specifically, mean activation time, activation dispersion, activation delay (i.e., duration of propagation from the earliest to latest region of the mapped area), and maximal activation time (i.e., longest activation time recorded in the mapped region) were improved by TRPV1 afferent depletion in MI + RTX subjects (Figure 4, D and E). Interestingly, RTX treatment had no significant impact on repolarization properties of the BZ tissue. Epicardial activation recovery intervals (ARIs, a surrogate for APD) from the scar BZ (Supplemental Figure 5, A and B) were not different between the 2 groups (consistent with AP data shown earlier in Figure 3, A-F), nor were ARI dispersion and ARI restitution properties of MI and MI + RTX subjects (Supplemental Figure 5, C and D).

Connexin $43(\mathrm{Cx} 43)$ distribution in the scar BZ is known to be reduced and lateralized following MI, and it is associated with VAs (20). We examined whether TRPV1 depletion also attenuated Cx43 remodeling in its reduction of postinfarct VAs. We observed restored $\mathrm{Cx} 43$ distribution in MI + RTX subjects in the scar BZ, while no significant differences were identified in myocardium remote to the infarcted region (Supplemental Figure 6). 
A

$\mathrm{MI}$
aVR

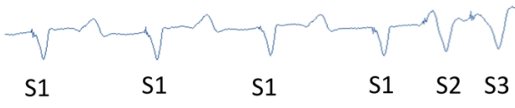

$\mathrm{aVL}$

\section{MI+RTX}

B

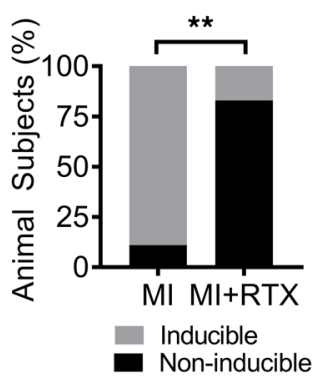

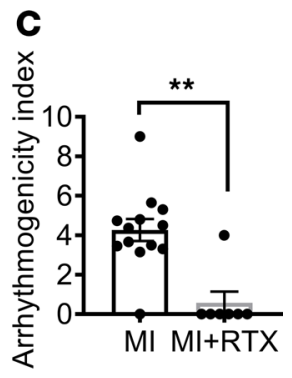

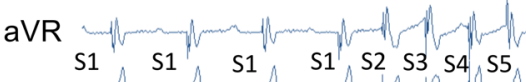

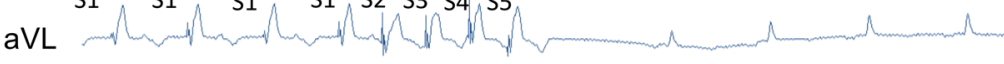

$500 \mathrm{~ms}$

D

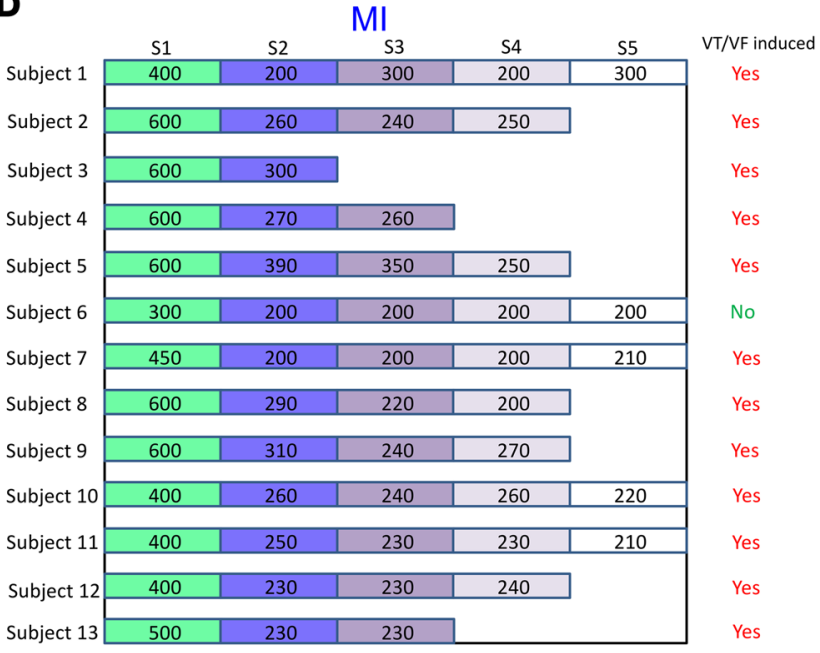

F Sinus Rhythm

P
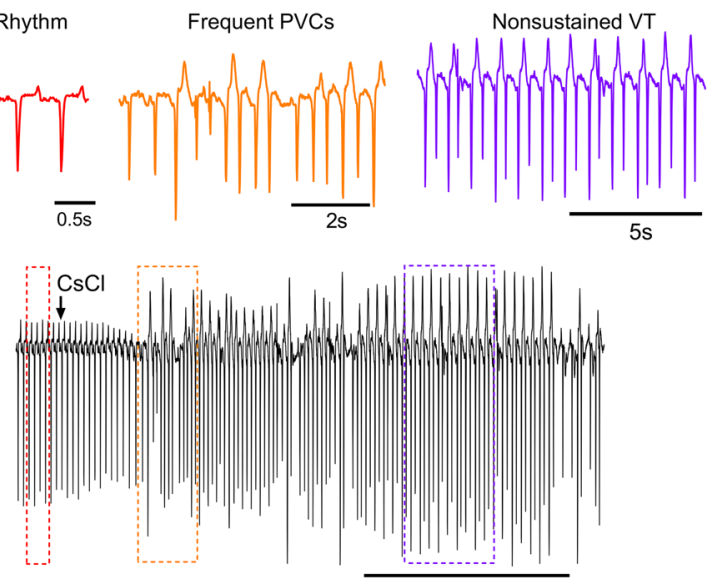

E

$\mathrm{Ml}+\mathrm{RTX}$

\begin{tabular}{|c|c|c|c|c|c|c|}
\hline \multirow[b]{2}{*}{ Subject 1} & S1 & S2 & S3 & S4 & S5 & \multirow{2}{*}{$\begin{array}{l}\text { VT/VF induced } \\
\text { No }\end{array}$} \\
\hline & 400 & 300 & 300 & 300 & 250 & \\
\hline \multirow{2}{*}{ Subject 2} & 350 & 200 & 200 & 200 & 200 & \multirow{2}{*}{ No } \\
\hline & & & & & & \\
\hline Subject 3 & 350 & 230 & 230 & 230 & 200 & No \\
\hline Subject 4 & 400 & 200 & 200 & 200 & 200 & Yes \\
\hline Subject 5 & 400 & 230 & 230 & 230 & 200 & No \\
\hline Subject 6 & 400 & 200 & 200 & 200 & 200 & No \\
\hline Subject 7 & 350 & 230 & 250 & 240 & 200 & No \\
\hline
\end{tabular}
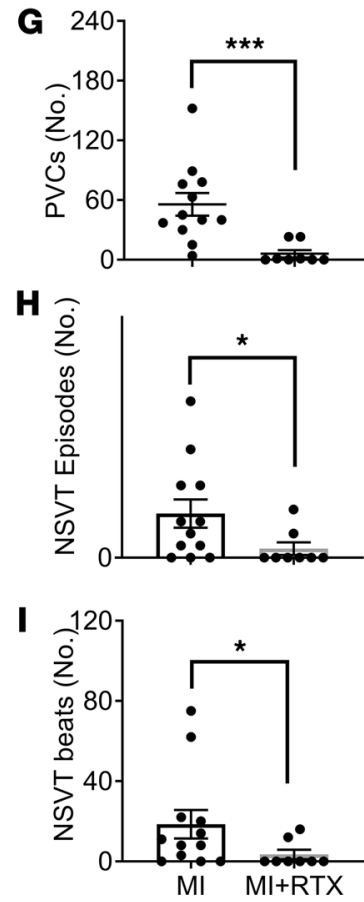

Figure 2. Cardiac sympathetic afferent depletion reduces ventricular arrhythmogenesis. (A) Representative tracing of ventricular tachycardia (VT) induced by programmed electrical stimulation in myocardial infarction (MI) with 2 extrastimuli (S3) but not in resiniferatoxin-treated (RTX-treated) MI subjects (MI + RTX) with 4 extrastimuli (S5). (B) Across subjects, VT induction was substantially reduced in MI + RTX subjects compared with MI $(P=0.005)$. (C-E) Arrhythmogenicity index, calculated from the drive cycle length and number of extrastimuli, was reduced in MI + RTX versus MI ( $n=13$ and 7 for MI and MI + RTX, respectively; $P=0.002)$. // for MI + RTX Subject 1 indicates ventricular effective refractory period. Ventricular arrhythmogenesis was also examined by bolus administration of cesium chloride $(80 \mathrm{mg} / \mathrm{kg}$ ). (F) Representative tracings showing sinus rhythm, frequent premature ventricular contractions (PVCs), and nonsustained ventricular tachycardia (NSVT) from an MI subject. (G-I) Reduction in number of PVCs, NSVT episodes, and number of beats of each NSVT episode in MI vs. MI + RTX animals ( $n=12$ and 8 for MI + RTX and MI, respectively). $\chi^{2}$ test (B) and Mann-Whitney $U$ test (C and $\left.\mathbf{G}-\mathbf{I}\right)$ were used. 
A

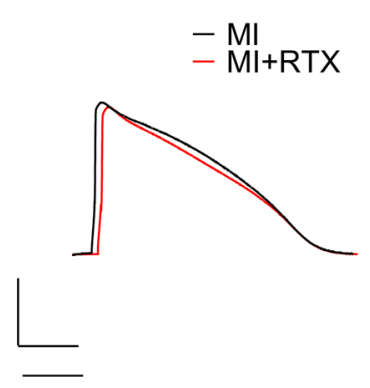

B

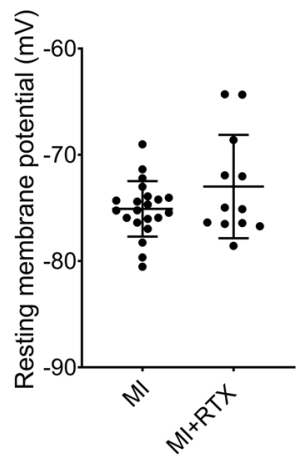

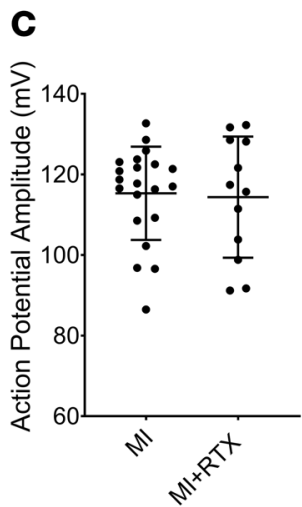

D

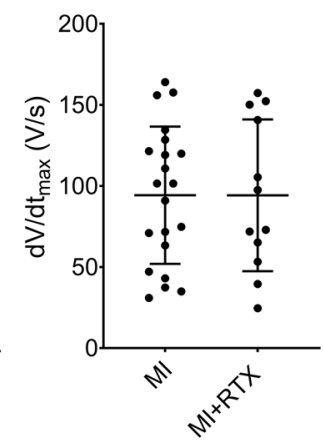

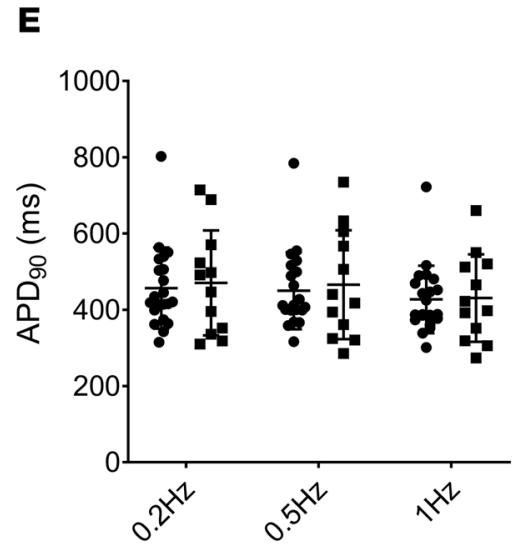

G

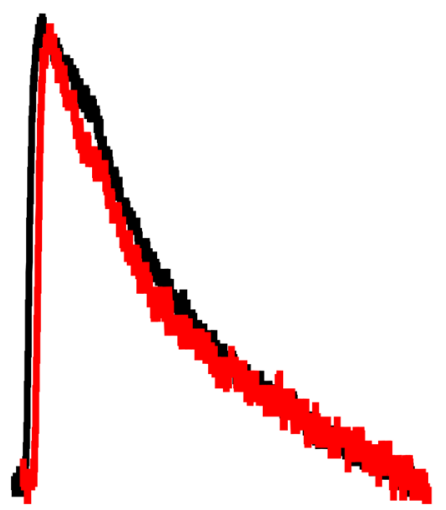

$\mathbf{F}$

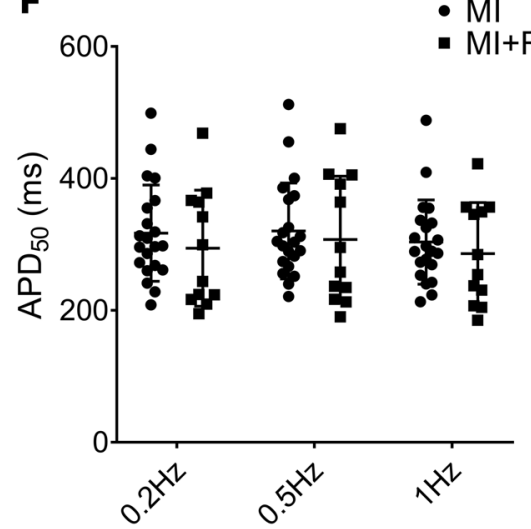

H

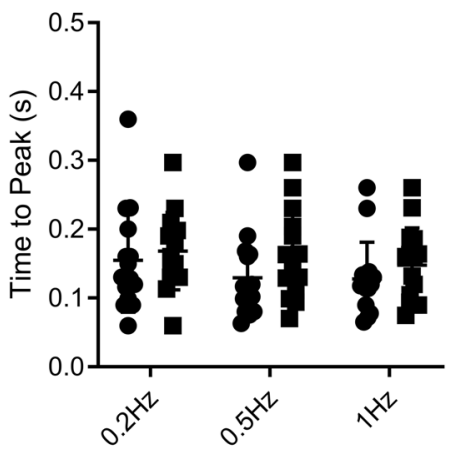

】

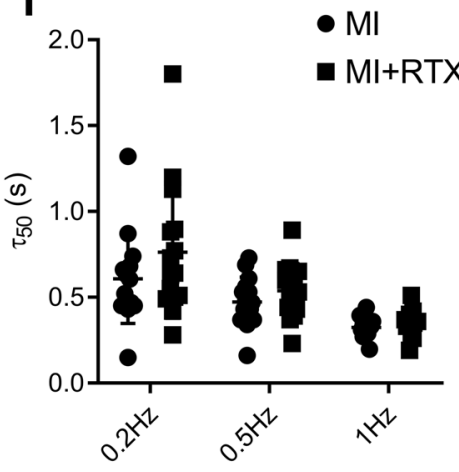

Figure 3. Cardiomyocyte electrophysiologic properties after cardiac TRPV1 afferent depletion. (A) Representative action potential (AP) tracing from untreated myocardial infarction (MI) and RTX-treated MI subjects (MI + RTX). (B-F) No differences were observed in resting membrane potential (B), AP amplitude $(\mathbf{C})$, rate of $A P$ rise $(\mathbf{D})$, and $A P D_{90}(\mathbf{E})$ and $A P D_{50}(\mathbf{F})$. (C) Representative tracing of calcium (C $\mathrm{C}^{2+}$ ) transients recorded from isolated border zone $\mathrm{MI}$ and $\mathrm{MI}+\mathrm{RTX}$ cardiomyocytes. ( $\mathbf{H}$ and $\mathbf{I})$ There were no differences in time to peak $\mathrm{Ca}^{2+}$ fluorescence $(\mathbf{H})$ and half maximal relaxation of the $\mathrm{Ca}^{2+}$ transient $\left(\tau_{50}\right)(\mathbf{I}) . n=15$ cells from 3 animals/group. Mann-Whitney $U$ test (B-D), 2-way ANOVA (H and I) were used.

In summary, these data suggest that cardiac TRPV1 afferent depletion exerts its antiarrhythmic effects by attenuating scar BZ fibrosis, $\mathrm{Cx} 43$ distribution, and activation heterogeneity at the tissue level - not by altering cellular AP or tissue repolarization properties.

Depletion of cardiac TRPV1 afferents preserves global cardiac function after MI. We sought to determine whether the structural changes mitigated by TRPV1 afferent depletion at the time of MI improved cardiac function as 
A

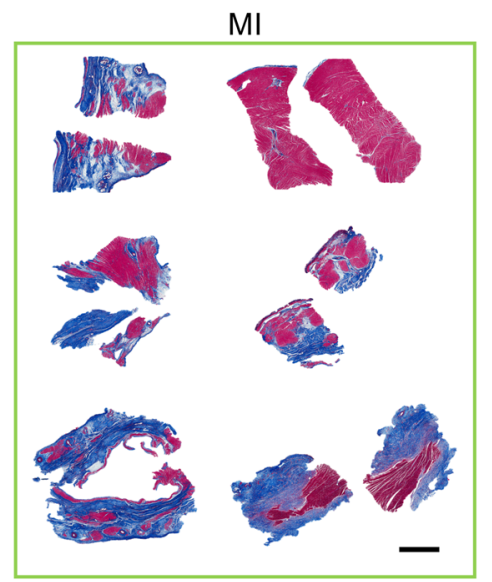

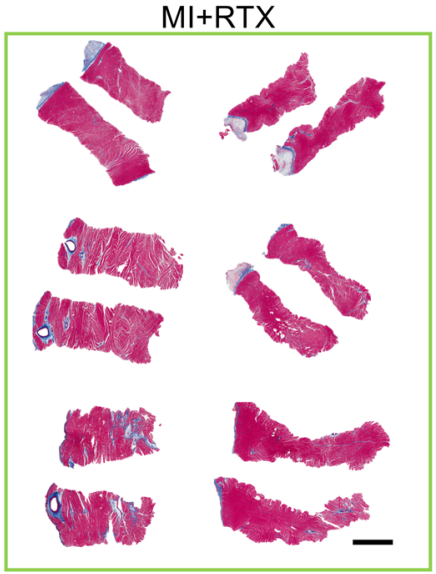

B
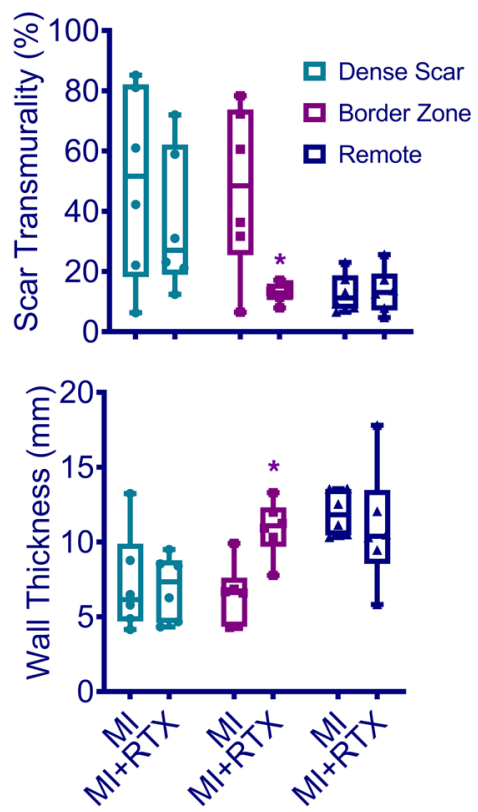

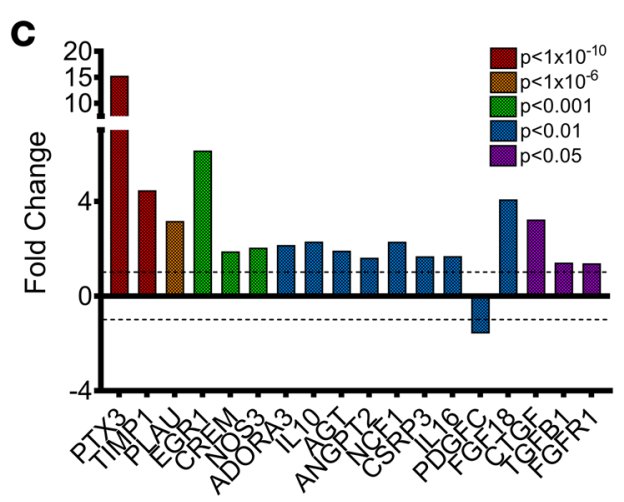

D

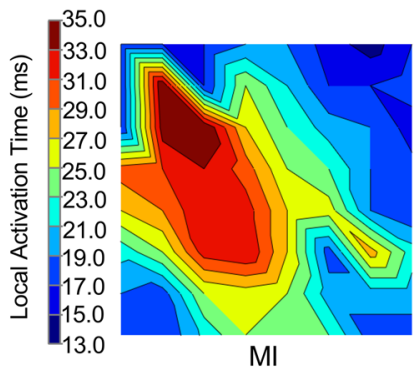

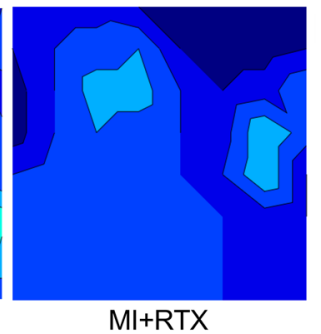

MI+RTX
E

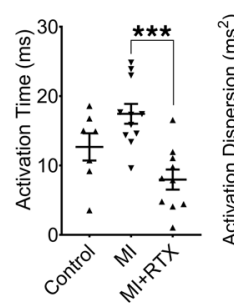

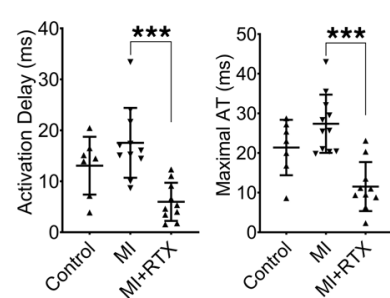

Figure 4. Cardiac TRPV1 afferent depletion ameliorates adverse postinfarction border zone remodeling. (A) Trichrome-stained transmural sections from the left ventricular (LV) border zone (BZ) of myocardial infarct (MI) subjects and the same region in resiniferatoxin-treated (RTX-treated) MI subjects (MI + RTX) show less scar (blue) in MI + RTX compared with MI. Scale bar: 2 mm. (B) Percent scar transmurality and wall thickness (top and bottom, respectively) in the dense scar region (apex), BZ, and remote myocardium (basal-lateral LV) from MI and MI + RTX animals show that the BZ, not dense scar or remote myocardium, is impacted by cardiac TRPV1 afferent depletion. (C) Several genes involved in fibrosis are differentially expressed in MI + RTX hearts compared with MI, as revealed by RNA sequencing of BZ myocardium. (D) Representative epicardial activation map in sinus rhythm from the scar BZ of MI and $\mathrm{MI}+\mathrm{RTX}$ subjects showing significant spatial activation heterogeneity in MI compared with MI + RTX. (E) Mean activation time, activation dispersion, activation delay, and maximal activation time in sham, MI, and MI + RTX subjects show reduced electrical heterogeneity in MI + RTX subjects. $n=10-11 /$ group. ${ }^{*} P<0.05 ;{ }^{* *} P<0.001$. ANOVA with Welch's test was used.

determined by echocardiography. MI resulted in reduced left ventricular ejection fraction (LVEF) and increased LV end-systolic volume (LVESV) and internal diameter in diastole (LVIDd), as well as LV wall thinning; however, treatment with RTX at the time of MI attenuated these changes (Figure 5, A-E).

Chronic cardiac TRPV1 afferent signaling promotes cardiac hyperdynamic adrenergic dysfunction. Since enhanced cardiac adrenergic tone results from cardiac TRPV1 afferent activation and is a powerful modulator of cardiac electrical function, we sought to examine whether sympathetic neuronal dysfunction (known to occur following MI) was the link between TRPV1 afferent activation and adverse BZ remodeling. This question would not be answered by $\beta A R$ or calcium channel blockade because, independent of arrhythmia mechanism, $\beta A R$ and calcium channel blockers are established antiarrhythmic agents. We turned to extracellular neuronal recordings 
A

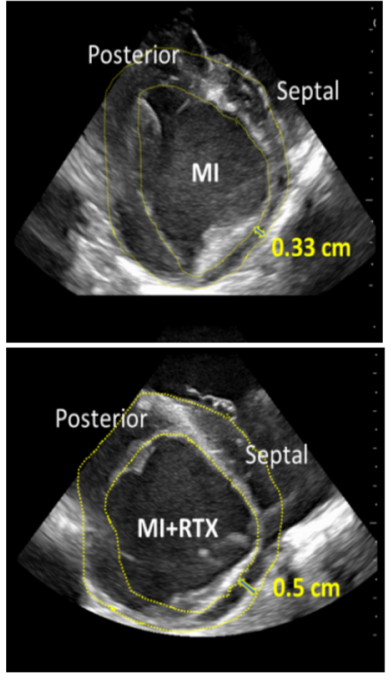

B

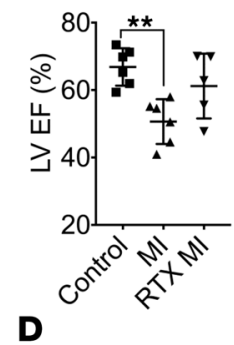

D

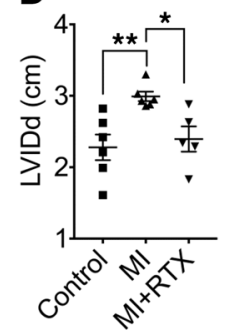

C

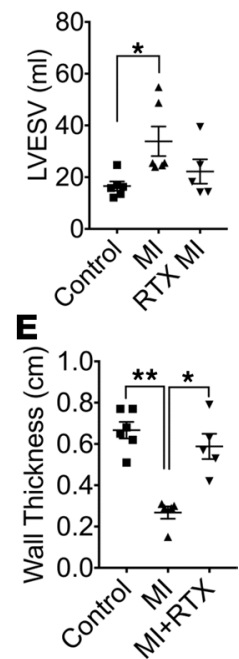

Figure 5. Cardiac TRPV1 depletion normalizes cardiac function after MI. (A) Representative left ventricular short axis echocardiographic images from MI and MI + RTX subjects. (B-E) Quantified data across subjects showing left ventricular (LV) ejection fraction (LVEF), LV end-systolic volume (LVESV), LV internal dimension in diastole (LVIDd), and LV anterior wall thickness, respectively $\left(n=5-6 /\right.$ group). ${ }^{*} P<0.05 ;{ }^{*} P<0.01$. Kruskall-Wallis test was used.

of sympathetic neurons located in the cardiac pole of the stellate ganglion, the major nexus point for efferent adrenergic outflow to the heart. We performed such recordings of stellate ganglion neurons (SGNs) in control, $\mathrm{MI}$, and MI + RTX animals. Filtered recordings were subjected to artifact removal and spike sorting using principal component analysis (PCA) and cluster on measurement analysis (Figure 6A). This identified the activity of individual neurons, which are designated as unique colors. A few examples are shown in Figure 6A. These approaches are detailed in the Supplemental Methods.

Basal SGN activity was greater in MI compared with control subjects (Figure 6B). Additionally, the proportion of neurons exhibiting spontaneous activity was greater in MI compared with control subjects (Figure 6C), indicating higher sympathetic tone after MI. Both indices of enhanced adrenergic tone were attenuated by cardiac TRPV1 afferent depletion (Figure 6, B and C). The responses of SGNs to cardiovascular stimuli were used to characterize reflex processing by SGNs (Figure 6D). SGN responses to cardiac mechanical stress (rapid ventricular pacing; RVP), afterload increase (aortic occlusion; Ao Occ), and preload reduction (inferior vena cava occlusion; IVC Occ) caused paradoxical changes in SGNs from MI compared with control subjects. TRPV1 afferent depletion in MI subjects (MI + RTX) largely restored these response patterns to those seen in control subjects. Conditional probability analysis $(21,22)$ was used to assess the degree of coordinated network function among cardiac-related SGNs to RVP, IVC Occ, and Ao Occ. It revealed a diminution in network processing capabilities of MI subjects compared with controls, and this was similarly normalized by cardiac TRPV1 afferent depletion (Figure 6E). These data indicate enhanced but uncoordinated reflex function of SGNs following MI; however, these adverse neural network processes were prevented by TRPV1 afferent depletion.

\section{Discussion}

The major findings of the present study are that chronic cardiac TRPV1 afferent signaling following MI promotes arrhythmogenic myocardium by driving deleterious myocardial remodeling and worsening sympathetic neuronal function. These accepted arrhythmogenic mechanisms act in concert to increase arrhythmia susceptibility in infarcted ventricles. To our knowledge, this represents the first reports of this phenomenon. A number of interrelated lines of evidence support this finding. First, cardiac TRPV1 afferent depletion by RTX significantly reduces inducible arrhythmias and conduction heterogeneity in the scar BZ without affecting basic electrophysiologic properties of BZ myocytes. Second, scar BZ fibrosis, expression of profibrotic genes in the scar BZ, and LV contractile dysfunction are ameliorated in RTX-treated animals. Third, enhanced and dysfunctional sympathetic neuronal function seen after MI are mitigated in 
A
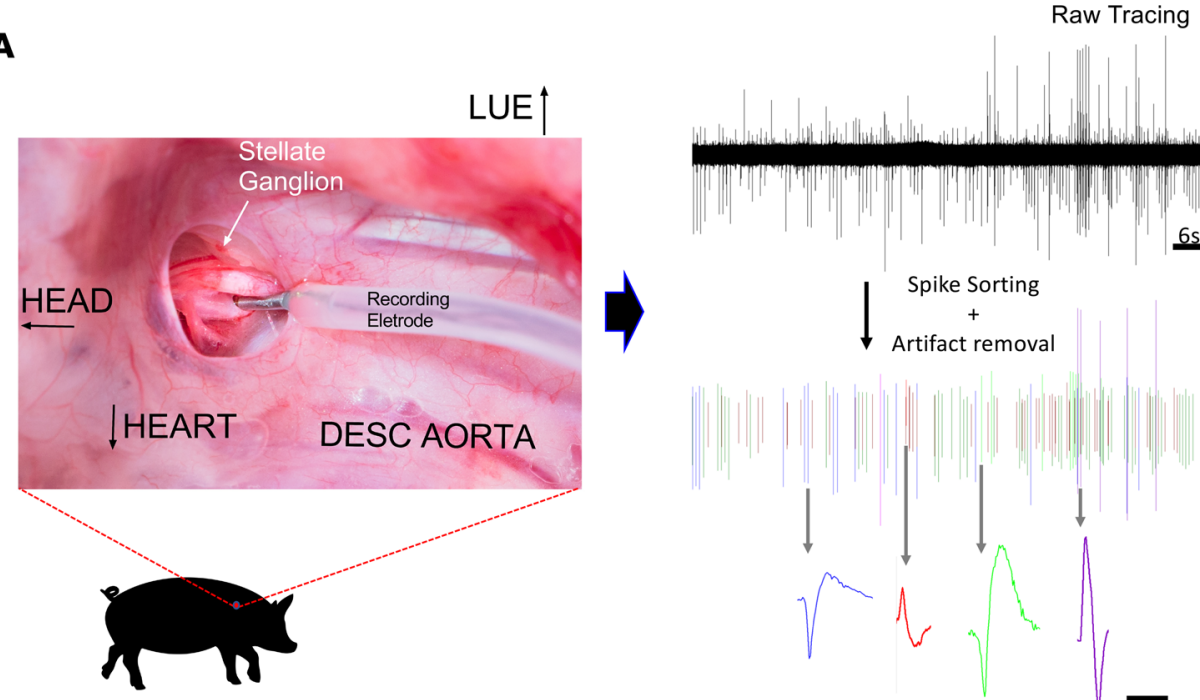

\$

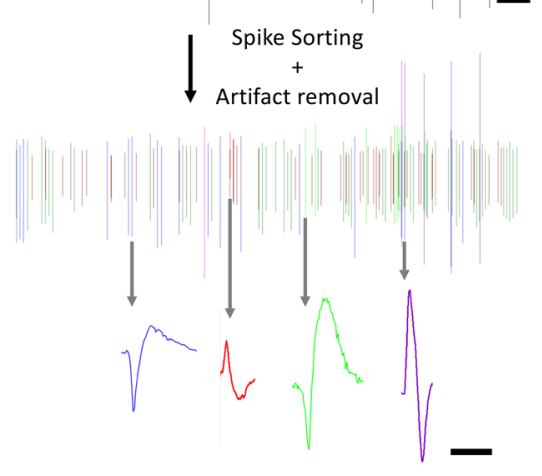

B

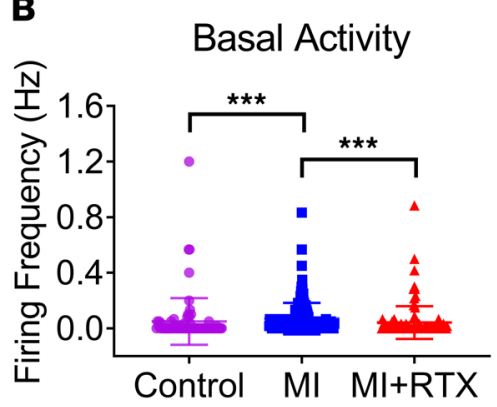

C

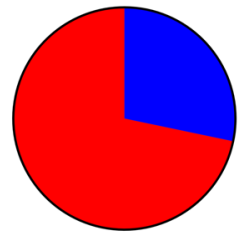

Control

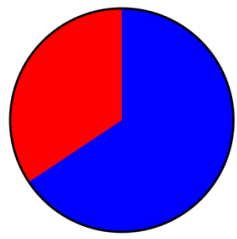

MI

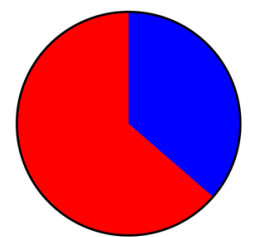

MI+RTX
Active Inactive

\section{D}

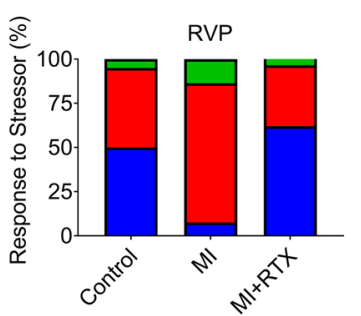

Decrease

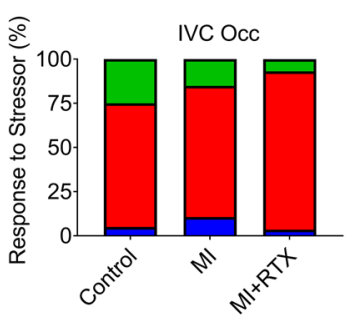

No Change

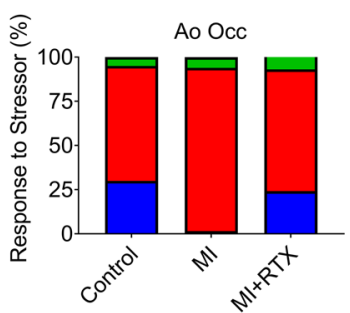

Increase

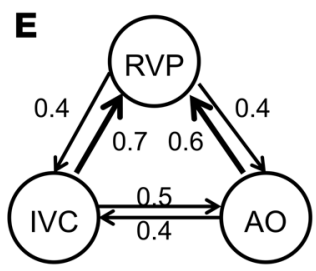

Control

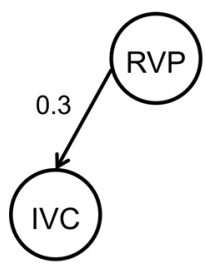

MI

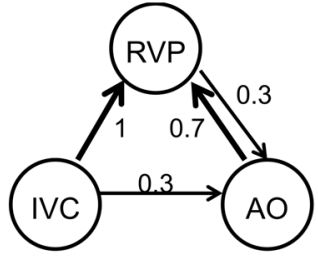

MI+RTX

Figure 6. Amelioration of infarct-induced sympathetic neuronal network dysfunction by cardiac TRPV1 afferent depletion. (A) Location of the recording electrode (left). DESC, descending; LUE, left upper extremity. Examples of spike-sorted neuronal action potential morphologies (right). Scale bar: 1 ms. (B) Basal activity of cardiovascular-related stellate ganglion neurons recorded from control, myocardial infarction (MI), and resiniferatoxin-treated (RTXtreated) MI subjects ( $\mathrm{MI}+\mathrm{RTX})$. MI subjects exhibited higher basal activity compared with control and $\mathrm{MI}+\mathrm{RTX}$ ( $n=78,137$, and 108 for control, $\mathrm{MI}$, and $\mathrm{MI}+\mathrm{RTX}$, respectively. ${ }^{* *} P<0.001$. ANOVA was used. (C) Of neurons recorded throughout the experimental protocol, more were active at baseline in MI subjects, compared with control and MI + RTX. $P<0.0001$. $\chi^{2}$ test was used; $n=22,90$, and 39, respectively. (D) Responses of recorded neurons to cardiovascular stressors: rapid ventricular pacing (RVP), inferior vena cava occlusion (IVC), and aortic occlusion (AO). Responses in MI subjects deviated from those in controls. However, responses in MI + RTX subjects were restored toward that seen in controls. (E) Conditional probability maps $(1,2)$ of the 3 stressors in $\mathbf{D}$, showing reduced network interdependence in MI compared with control, and reversion toward normal physiology in MI + RTX subjects. 
RTX-treated subjects. Taken together, these findings suggest that chronic TRPV1 afferent signaling plays an important role in the pathophysiologic remodeling of infarcted tissues that confers arrhythmogenicity.

Prior studies have suggested that, while elimination of cardiac sympathetic innervation after MI does not reduce infarct size, it substantially reduced myocardial inflammation, myocyte hypertrophy, and cardiac dysfunction (23). Similar findings were observed in a pressure overload model, where interstitial fibrosis was reduced by elimination of cardiac sympathetic nerves (24). Although this influence exerted by cardiac sympathetic innervation was known, drivers of enhanced sympathetic activity remain poorly understood. We investigated the role of cardiac TRPV1 afferent signaling in this paradigm (Supplemental Figure 7) and identified it as a key driver of enhanced adrenergic function and adverse myocardial remodeling following MI.

The findings of the present study are distinct from those in a rodent $\mathrm{KO}$ model of the TRPV1 channel, where pressure overload-induced cardiac hypertrophy was exacerbated by the genetic ablation of the TRPV1 channel $(25,26)$. These findings are not surprising, given the important role TRPV1 afferents play in the early disease process, where they mediate the protective responses to ischemia, as an example. As a result, genetic ablation of this channel offers no protective mechanism to ischemia and cardiac stress. Chronically, however, continued TPRV1 afferent signaling promotes adrenergic drive to the heart, along with its maladaptive consequences. In the chronic setting, elimination of TRPV1 afferents is beneficial but not acutely. In our study, the mechanism by which RTX eliminates TRPV1 afferent fibers (toxic excitation) enables the acute beneficial responses of cardiac TRPV1 afferents; however, over the ensuing days, it leads to elimination of the afferent fibers. This key difference between RTX-mediated depletion of TRPV1 afferents and genetic ablation of the TRPV1 channel is critical to differentiating the findings of our study from rodent $\mathrm{KO}$ studies. Our findings are supported by MI studies in rodents treated with RTX, in which fibrosis and cardiac function was improved to a similar degree as in our porcine study, and in contrast with rodent genetic ablation studies.

Our study also highlights dysfunctional reflex processing with the stellate ganglion following MI. The stellate ganglion is a major nexus point in cardiac adrenergic signaling as a critical site for pre- to postganglionic synapses. However, increasingly, the stellate ganglion is recognized to have processing capabilities. The degree to which there is dysfunctional processing following MI was not well understood. In the present study, we show that $\mathrm{MI}$ is associated with increased neuronal firing rates and a loss of coordinated network function in stellate ganglia. Interestingly, this loss of coordination after MI was partially attenuated by cardiac TRPV1 afferent depletion, suggesting a role for these afferent fibers in neuronal dysfunction after MI. This highlights another pathophysiologic mechanism linking adrenergic dysfunction with cardiac injury. Importantly — while we have shown an association between chronic hyperdynamic, uncoordinated adrenergic function, and adverse remodeling after MI - these data do not specifically implicate adrenergic hyperactivation in the initiation of VT/VF.

Study limitations. TRPV1 channels have been reported in cardiomyocytes, although the intracellular location remains controversial $(27,28)$. It is unlikely that myocardial TRPV1 modulation influenced our findings because (a) experiments were performed 4 weeks after RTX was applied and (b) the expected effect of RTX activation of the TRPV1 channel is an irreversible calcium influx, which would result in cell death. This did not occur, as verified by histology, LV function (assessed by echocardiography), and ex vivo high-resolution gadolinium-enhanced cardiac MRI (1 mm voxel resolution) in normal hearts. Toxic irreversible activation of TRPV1 in cardiomyocytes would also worsen LV function after MI, instead of improving it, as shown in the present study (Supplemental Figure 7).

Delivery of RTX required percutaneous epicardial access, which may limit clinical translation of this approach. However, percutaneous epicardial access is increasingly used for ablation and left atrial appendage closure. Genetic ablation of TRPV1 may further clarify our findings; however, such models do not exist in large animals. Although porcine subjects more closely recapitulate human pathophysiology than murine models, some caution should be exercised in extrapolating these findings to humans, given some resistance to $\beta \mathrm{AR}$ blockers in preventing VT/VF during acute ischemia in 1 porcine study (29).

Conclusions. These data in composite demonstrate that epicardial RTX application in the early ischemic period is a feasible method to deplete TRPV1 sensory afferents. These afferent fibers chronically drive sympathoexcitation following MI, promoting adverse myocardial remodeling, electrical heterogeneity, and VT/VF risk (Supplemental Figure 7). Cardiac-restricted targeting of chronic CSAR ameliorates these pathologic processes and renders infarcted hearts more stable and resistant to VAs. This implicates TRPV1 afferents as key targets in reducing post-MI cardiac dysfunction and arrhythmias, to which novel minimally or noninvasive approaches can be developed. 


\section{Methods}

Supplemental Methods are available online with this article.

Animal models and protocols. Yorkshire pigs $(n=39)$ with normal hearts (control; $n=11 ; 50.89 \pm 1.66$ $\mathrm{kg})$, and chronic anterior MI $(n=16 ; 52.04 \pm 1.91 \mathrm{~kg})$ were compared with RTX-treated pigs with chronic anterior MI (MI + RTX; $n=12 ; 50.62 \pm 3.87 \mathrm{~kg}$ ) (S\&S Farms). Each subject was sedated with terconazole (4-8 $\mathrm{mg} / \mathrm{kg}$, intramuscular), intubated, and placed under general anesthesia. Inhaled isoflurane (2\%) with supplemental oxygen ( $2 \mathrm{~L} /$ minute) during the experimental study. Surface electrocardiogram (ECG) and arterial blood pressure (ABP) were continuously monitored.

$R T X$ administration. Isoflupredone acetate $(0.04 \mathrm{mg} / \mathrm{kg})$ and famotidine $(0.5 \mathrm{mg} / \mathrm{kg})$ were given intramuscularly 24 hours prior to RTX administration. The left sternocostal angle was anesthetized with $1 \%$ lidocaine before making a $0.5-1.0 \mathrm{~cm}$ incision. Percutaneous access to the intrapericardial space was obtained using a Tuohy needle under fluoroscopic observation (14). A small amount of contrast agent was used to ensure the advancement of the needle tip, and intrapericardial access was confirmed by cardiac ectopy and tension before entering the pericardial space. Access was confirmed by a short J-tip guidewire taking a cardiac border-forming configuration fluoroscopically. The incision was progressively dilated with 6- to 8-Fr dilators. A multipurpose sheath was then advanced over the J-tip wire in order to aspirate pericardial fluid and administer $10 \mathrm{~mL}$ of RTX $(12.5 \mu \mathrm{g} / \mathrm{mL})$. Twenty minutes later, RTX was aspirated, and methylprednisolone sodium succinate $(50 \mathrm{mg}$ ) was injected to prevent pericardial adhesions. Buprenorphine $(0.02 \mathrm{mg} / \mathrm{kg})$ was administered intramuscularly starting 6 hours after the procedure and then every 24 hours for 2 days. Carprofen $(4 \mathrm{mg} / \mathrm{kg}$ ) and famotidine (20 mg/day) were also orally given every 24 hours for 3 days after procedure. In sham subjects, the protocol with repeated identically, with saline vehicle infused instead.

Isolation of BZ ventricular myocytes. Following euthanasia, BZ myocardium (adjacent but distinct from dense scar) was rapidly biopsied and $0.8 \mathrm{~cm}^{3}$ samples were placed in storage solution as described by Workman et al. (30). We then performed collagenase/protease isolation of ventricular myocytes as described (31). Myocytes were stored at room temperature in Kraft-Bruhe solution ( $\mathrm{pH} 7.4$ ) until use within 4 hours of isolation.

$A P$ recordings. For AP recordings, we placed isolated cardiomyocytes in an experimental chamber $(0.5$ $\mathrm{mL}$ ) mounted on the stage of a Nikon Diaphot inverted microscope modified for simultaneous patch clamping and cellular epifluorescence recording. A modified Tyrode bath solution (in mmol/L: $\mathrm{NaCl} \mathrm{136,} \mathrm{KCl}$ 5.4, $\mathrm{NaH}_{2} \mathrm{PO}_{4} 0.33, \mathrm{MgCl}_{2} 1.0$, HEPES $10, \mathrm{CaCl}_{2} 1.8$, glucose $10, \mathrm{pH} 7.4$ ) continuously perfused the chamber. Patch electrodes were pulled from borosilicate glass (TW150F-3, World Precision Instruments) on a horizontal puller (P-97, Sutter Instruments). The fire-polished electrodes had a tip resistance of 1-2 M 2 . The internal solution contained (in mmol/L) $\mathrm{NaCl} 10, \mathrm{KCl} 130$, HEPES 10, MgATP 5, phosphocreatine 5, cAMP 0.05, and $\mathrm{MgCl}_{2}$ 1, pH adjusted to 7.2 with $\mathrm{KOH}$. We used an Axopatch 200B patch-clamp amplifier (Molecular Devices) under the control of pCLAMP 10 software (Molecular Devices) and a Digidata 1440A PC interface (Molecular Devices) to induce and record APs in whole-cell current clamp mode.

Measurement of $\mathrm{Ca}^{2+}$ transients. We loaded isolated cardiomyocytes with the $\mathrm{Ca}^{2+}$ indicator Fluo- 4 by incubating cells in bath solution containing Fluo-4 AM $(10 \mu \mathrm{M})$ and Pluronic F-127 (0.02\%) for 30 minutes, followed by 3 washes in dye-free bath solution for 5 minutes each. The loading and washout times were sufficient for deesterification of the dye. We then recorded Fluo-4 fluorescence signals using a photodiode during simultaneous current clamp with a custom-designed photometric epifluorescence detection system attached to the patch clamp microscope described above. We used a $488 \mathrm{~nm}$ LED light source for excitation and a longpass filter to restrict emission to $>510 \mathrm{~nm}$. Fluorescence ratios were normalized to baseline $\left(\mathrm{F} / \mathrm{F}_{0}\right)$ after background subtraction.

Statistics. Variables are presented as means \pm SEM. The Shapiro-Wilk test was used for assessing distribution. A 2-tailed Student's $t$ test and 2-way ANOVA test were used for data that were normally distributed, and the Mann-Whitney $U$ test and Kruskal-Wallis test were used for data that were not normally distributed. VT inducibility in MI vs MI + RTX subjects were analyzed using the $\chi^{2}$ test. ${ }^{*} P<0.05 ;{ }^{* *} P<0.01 ;{ }^{* *} P$ $<0.001 ;{ }^{* * * *} P<0.0001$. Analysis was performed using Microsoft Excel and Graphpad Prism.

Study approval. Studies were performed in accordance with the University of California Institutional Animal Care and Use Committee (IACUC) guideline and the NIH Guide for the Care and Use of Laboratory Animals (National Academies Press, 2011). 


\section{Author contributions}

KY, JIG, and OAA designed research studies. KY, PSR, LM, JM, MAS, XW, RZ, and OAA conducted experiments and acquired data. KY, LM, JM, TS, RZ, and SS analyzed data. KY, SS, and OAA wrote the manuscript, and all authors approved the manuscript.

\section{Acknowledgments}

The authors thank Amiksha Gandhi, OhJin Kwon, Christian Wooten, and Yuichi Hori for excellent technical assistance, and Jeffrey Ardell and Kalyanam Shivkumar for comments on the manuscript. This study was supported by an AIBA WORKS basic medical research fellowship to KY, and by NIH grants HL125730, OT2OD023848, and DP2HL142045 to OAA. Cardiac electrophysiologic mapping arrays were supported by U01EB025138.

Address correspondence to: Olujimi A. Ajijola, UCLA Cardiac Arrhythmia Center, UCLA Neurocardiology Research Center, David Geffen School of Medicine at UCLA, 100 Medical Plaza, Suite 660, Los Angeles California 90095, USA. Phone: 310.206.6433; Email: oajijola@mednet.ucla.edu.

1. Ardell JL, et al. Translational neurocardiology: preclinical models and cardioneural integrative aspects. J Physiol (Lond). 2016;594(14):3877-3909.

2. Shivkumar K, et al. Clinical neurocardiology defining the value of neuroscience-based cardiovascular therapeutics. $J$ Physiol (Lond). 2016;594(14):3911-3954

3. O'Gara PT, et al. 2013 ACCF/AHA guideline for the management of ST-elevation myocardial infarction: executive summary: a report of the American College of Cardiology Foundation/American Heart Association Task Force on Practice Guidelines: developed in collaboration with the American College of Emergency Physicians and Society for Cardiovascular Angiography and Interventions. Catheter Cardiovasc Interv. 2013;82(1):E1-27.

4. Brown AM. Excitation of afferent cardiac sympathetic nerve fibres during myocardial ischaemia. J Physiol (Lond) 1967;190(1):35-53.

5. Malliani A, Schwartz PJ, Zanchetti A. A sympathetic reflex elicited by experimental coronary occlusion. Am J Physiol. 1969;217(3):703-709.

6. Malliani A, Recordati G, Schwartz PJ. Nervous activity of afferent cardiac sympathetic fibres with atrial and ventricular endings. J Physiol (Lond). 1973;229(2):457-469.

7. Wang W, Schultz HD, Ma R. Cardiac sympathetic afferent sensitivity is enhanced in heart failure. Am J Physiol. 1999;277(2):H812-H817.

8. Zahner MR, Li DP, Chen SR, Pan HL. Cardiac vanilloid receptor 1-expressing afferent nerves and their role in the cardiogenic sympathetic reflex in rats. J Physiol (Lond). 2003;551(Pt 2):515-523.

9. Caterina MJ, Schumacher MA, Tominaga M, Rosen TA, Levine JD, Julius D. The capsaicin receptor: a heat-activated ion channel in the pain pathway. Nature. 1997;389(6653):816-824.

10. Karai L, et al. Deletion of vanilloid receptor 1-expressing primary afferent neurons for pain control. J Clin Invest. 2004;113(9):1344-1352.

11. Wang HJ, Wang W, Cornish KG, Rozanski GJ, Zucker IH. Cardiac sympathetic afferent denervation attenuates cardiac remodeling and improves cardiovascular dysfunction in rats with heart failure. Hypertension. 2014;64(4):745-755.

12. Yoshie K, et al. Cardiac vanilloid receptor-1 afferent depletion enhances stellate ganglion neuronal activity and efferent sympathetic response to cardiac stress. Am J Physiol Heart Circ Physiol. 2018;314(5):H954-H966.

13. Price TJ, Flores CM. Critical evaluation of the colocalization between calcitonin gene-related peptide, substance $P$, transient receptor potential vanilloid subfamily type 1 immunoreactivities, and isolectin B4 binding in primary afferent neurons of the rat and mouse. J Pain. 2007;8(3):263-272.

14. Lawson SN, Perry MJ, Prabhakar E, McCarthy PW. Primary sensory neurones: neurofilament, neuropeptides, and conduction velocity. Brain Res Bull. 1993;30(3-4):239-243.

15. Marban E, Robinson SW, Wier WG. Mechanisms of arrhythmogenic delayed and early afterdepolarizations in ferret ventricular muscle. J Clin Invest. 1986;78(5):1185-1192.

16. Pinto JM, Boyden PA. Electrical remodeling in ischemia and infarction. Cardiovasc Res. 1999;42(2):284-297.

17. Dillon SM, Allessie MA, Ursell PC, Wit AL. Influences of anisotropic tissue structure on reentrant circuits in the epicardial border zone of subacute canine infarcts. Circ Res. 1988;63(1):182-206.

18. Landstrom AP, Dobrev D, Wehrens XHT. Calcium Signaling and Cardiac Arrhythmias. Circ Res. 2017;120(12):1969-1993.

19. Ajijola OA, et al. Sympathetic modulation of electrical activation in normal and infarcted myocardium: implications for arrhythmogenesis. Am J Physiol Heart Circ Physiol. 2017;312(3):H608-H621.

20. Peters NS, Coromilas J, Severs NJ, Wit AL. Disturbed connexin43 gap junction distribution correlates with the location of reentrant circuits in the epicardial border zone of healing canine infarcts that cause ventricular tachycardia. Circulation. 1997;95(4):988-996.

21. Beaumont E, et al. Network interactions within the canine intrinsic cardiac nervous system: implications for reflex control of regional cardiac function. J Physiol (Lond). 2013;591(18):4515-4533.

22. Rajendran PS, et al. Myocardial infarction induces structural and functional remodelling of the intrinsic cardiac nervous system. J Physiol (Lond). 2016;594(2):321-341. 
23. Ziegler KA, Ahles A, Wille T, Kerler J, Ramanujam D, Engelhardt S. Local sympathetic denervation attenuates myocardial inflammation and improves cardiac function after myocardial infarction in mice. Cardiovasc Res. 2018;114(2):291-299.

24. Perlini S, et al. Sympathectomy or doxazosin, but not propranolol, blunt myocardial interstitial fibrosis in pressure-overload hypertrophy. Hypertension. 2005;46(5):1213-1218.

25. Zhong B, Rubinstein J, Ma S, Wang DH. Genetic ablation of TRPV1 exacerbates pressure overload-induced cardiac hypertrophy. Biomed Pharmacother. 2018;99:261-270.

26. Huang W, Rubinstein J, Prieto AR, Thang LV, Wang DH. Transient receptor potential vanilloid gene deletion exacerbates inflammation and atypical cardiac remodeling after myocardial infarction. Hypertension. 2009;53(2):243-250.

27. Andrei SR, Sinharoy P, Bratz IN, Damron DS. TRPA1 is functionally co-expressed with TRPV1 in cardiac muscle: Co-localization at z-discs, costameres and intercalated discs. Channels (Austin). 2016;10(5):395-409.

28. Hurt CM, et al. Transient Receptor Potential Vanilloid 1 Regulates Mitochondrial Membrane Potential and Myocardial Reperfusion Injury. J Am Heart Assoc. 2016;5(9):e003774.

29. Skinner JE, Lie JT, Entman ML. Modification of ventricular fibrillation latency following coronary artery occlusion in the conscious pig. Circulation. 1975;51(4):656-667.

30. Workman AJ, Kane KA, Rankin AC. The contribution of ionic currents to changes in refractoriness of human atrial myocytes associated with chronic atrial fibrillation. Cardiovasc Res. 2001;52(2):226-235.

31. Reuter H, Han T, Motter C, Philipson KD, Goldhaber JI. Mice overexpressing the cardiac sodium-calcium exchanger: defects in excitation-contraction coupling. J Physiol (Lond). 2004;554(Pt 3):779-789. 